\title{
Pion condensation phase at finite isospin chemical potential in a Holographic QCD model
}

\author{
Hiroki Nishihara ${ }^{* a}$ and Masayasu Harada, ${ }^{a}$ \\ ${ }^{a}$ Department of Physics, Nagoya University, Nagoya 464-8602, Japan \\ E-mail: h248raehken.phys.nagoya-u.ac.jp \\ harada@hken.phys.nagoya-u.ac.jp
}

\begin{abstract}
We summarize main points of our recent work where we studied the pion condensation for nonzero isospin chemical potential within a holographic QCD model. We confirmed that the second order phase transition with the mean field exponent occurs when the isospin chemical potential $\mu_{I}$ exceed the pion mass, which is consistent with the result obtained by the chiral effective Lagrangian at $O\left(p^{2}\right)$. We find that a deviation for large $\mu_{I}$ region can be understood as a higher order effects in the chiral effective Lagrangian. Our result shows that the chiral condensate defined by $\tilde{\sigma} \equiv \sqrt{\langle\sigma\rangle^{2}+\left\langle\pi^{a}\right\rangle^{2}}$ is almost constant in the small $\mu_{I}$ region, while it grows with $\mu_{I}$ in the large $\mu_{I}$ region showing an enhancement of the chiral symmetry breaking.
\end{abstract}

XV International Conference on Hadron Spectroscopy

4-8/11/2013

Nara, Japan

\footnotetext{
* Speaker.
} 


\section{Introduction}

In this write-up, we summarize main points of the analysis done in Ref. [1], where we studied the pion condensation phase at finite chemical potential using a holographic QCD model [2].

Studying QCD at finite isospin chemical potential will give a clue to understand the symmetry energy which is important to describe the equation of state inside neutron stars. In addition, it may give some informations on the chiral symmetry structure of QCD. When we turn on the isospin chemical potential $\mu_{I}$ at zero baryon number density, the pion condensation is expected to occur at a critical point. It was shown [3] showed that, by using the chiral Lagrangian at $O\left(p^{2}\right)$, the second order phase transition to the pion condensation phase occurs and the critical value of $\mu_{I}$ is equal to the pion mass. There are so many works on the pion condensation at finite isospin chemical potential, there are not many works for studying the strength of the chiral symmetry breaking. Namely, it is interesting to ask whether or not the chiral symmetry is partially restored in the isospin matter.

In Ref. [1], we study the pion condensation phase in a holographic QCD model [2] by introducing the mean fields for $\pi, \sigma$ and the time component of $\rho$ meson. Our results show that the phase transition is of the second order consistently with the one obtained in the $O\left(p^{2}\right)$ chiral Lagrangian [3]. It is remarkable that the chiral condensate defined by $\tilde{\sigma} \equiv \sqrt{\langle\sigma\rangle^{2}+\left\langle\pi^{a}\right\rangle^{2}}$ is almost constant in the small $\mu_{I}$ region, while it grows with $\mu_{I}$ in the large $\mu_{I}$ region. This implies that the chiral symmetry breaking is enhanced by the existence of the isospin chemical potential.

\section{Model}

In the present analysis we use the hard-wall holographic QCD model given in Ref. [2]. The action is given by

$$
S_{5}=\int d^{4} x \int_{\varepsilon}^{z_{m}} d z \sqrt{g} \operatorname{Tr}\left[|D X|^{2}+3|X|^{2}-\frac{1}{4 g_{5}^{2}}\left(F_{L}^{2}+F_{R}^{2}\right)\right]+\mathscr{L}_{5}^{B D}
$$

where the metric is $d s^{2}=a^{2}(z)\left(\eta_{\mu \nu} d x^{\mu} d x^{\nu}-d z^{2}\right)$ with $a(z)=1 / z$ and the fifth direction $z$ has the UV and the IR cutoffs, $\varepsilon$ and $z_{m}$. This theory has the chiral symmetry $\mathrm{U}(2)_{\mathrm{L}} \times \mathrm{U}(2)_{\mathrm{R}}$, and that is consisted by a scalar field $X$ and the gauge fields corresponding to the chiral symmetry $\mathrm{U}(2)_{\mathrm{L}} \times \mathrm{U}(2)_{\mathrm{R}}$ The action contains the IR boundary term $\mathscr{L}_{5}^{B D}$ and the profile of $\mathscr{L}_{5}^{B D}$ is the $\phi$ fourth type of the $X$ field which has two parameters $\lambda$ and $m^{2}$. In the following analysis we adopt the $L_{5}=R_{5}=0$ gauge, and use the IR-boundary condition $\left.F_{5 \mu}^{L}\right|_{z_{m}}=\left.F_{5 \mu}^{R}\right|_{z_{m}}=0$.

In the vacuum the chiral symmetry is spontaneously broken down to $\mathrm{U}(2)_{V}$ by the vacuum expectation value of $X$. This is given by solving equation of motion and the solution of the $X$ has two parameters $m_{q}$ and $\sigma$, where $m_{q}$ corresponds to the current quark mass and $\sigma$ to the quark condensate [2]. They are related with $\lambda$ and $m^{2}$ by the IR-boundary condition of the $X$. A parameter $g_{5}^{2}$ is determined by matching with QCD as $g_{5}^{2}=\frac{12 \pi^{2}}{N_{c}}$. The pion is described as a linear combination of the lowest eigenstate of $\pi^{a}$ and the longitudinal mode of $A_{\mu}^{a}$, and the $\rho$ meson is the lowest eigenstate of $V_{\mu}$. The values of the $m_{q}$ and $z_{m}$ together with that of the relation between these parameters $\lambda$ and $m^{2}$ are fixed by fitting them to the pion mass $m_{\pi}=139.6 \mathrm{MeV}$, the $\rho$ meson mass $m_{\rho}=775.8$ $\mathrm{MeV}$ and the pion decay constant $f_{\pi}=92.4 \mathrm{MeV}: m_{q}=2.29 \mathrm{MeV}, z_{m}=1 /(323 \mathrm{MeV})$. In the 
present analysis, we use the $a_{0}$ meson mass $m_{a_{0}}=980 \mathrm{MeV}$ as a reference value, which fixes $m^{2}=5.39$ and $\lambda=4.4$, and see the dependence of our results on the scalar meson mass.

\section{Pion condensation phase}

In this section we study the pion condensation for finite isospin chemical potential $\mu_{I}$ in the holographic QCD model introduced in section 2

The isospin chemical potential $\mu_{I}$ is introduced as a UV-boundary value of the time component of the gauge field of $\mathrm{SU}(2)_{\mathrm{V}}$ symmetry as

$$
\left.V_{0}^{3}(z)\right|_{\varepsilon}=\mu_{I}
$$

where the superscript 3 indicates the third component of the isospin corresponding to the neutral $\rho$ meson. Here we study the pion condensation phase for small $\mu_{I}$, then we assume that the rotational symmetry $\mathrm{O}(3)$ is not broken by e.g. the $\rho$ meson condensation: $L_{i}=R_{i}=0$. We also assume the time-independent condensate, then the vacuum structure is determined by studying the mean fields of five-dimensional fields which do not depend on the four-dimensional coordinate. Furtheremore, we also take the mean fields for the neutral pion, the iso-triplet scalar meson ( $a_{0}$ meson) and the iso-singlet pseudoscalar meson ( $\eta$ meson) to be zero in the pion condensation phase .

We show the resultant relation between $\mu_{I}$ and the isospin density in Fig. 1 for $\lambda=1,4.4$ and 100 corresponding to $m_{a_{0}}=610 \mathrm{MeV}, 980 \mathrm{MeV}$ and $1210 \mathrm{MeV}$. This shows that the phase transition

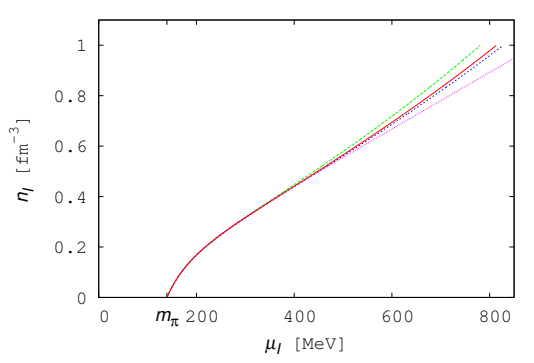

Figure 1: Relation between the isospin number density $n_{I}$ and the isospin number chemical potential $\mu_{I}$. The green, red and blue curves show our results for $\lambda=1,4.4$ and 100, respectively. The pink dashed-curve shows the result given by the chiral Lagrangian in Ref. [3]. Each choice of $\lambda$ corresponds to $m_{a_{0}}=610 \mathrm{MeV}$, $980 \mathrm{MeV}$ and $1210 \mathrm{MeV}$, respectively.

is of the second order and the critical chemical potential is predicted to be equal to the pion mass. This is consistent with the result obtained by the chiral Lagrangian approach in Ref. [3]. Furthermore, our result on the relation between isospin number density and isospin chemical potential for small $\mu_{I}$ agrees with the following one obtained by $\mathrm{O}\left(p^{2}\right)$ chiral Lagrangian [3]:

$$
n_{I}=f_{\pi}^{2} \mu_{I}\left(1-\frac{m_{\pi}^{4}}{\mu_{I}^{4}}\right)
$$

For $\mu_{I}>500 \mathrm{MeV}$, there is a difference between our predictions and the one from $\mathrm{O}\left(p^{2}\right)$ chiral Lagrangian, which can be understood as the higher order contribution as we will show in the next section. 
We show the $\mu_{I}$ dependences of these condensate in Fig. 2 , where $\langle\sigma\rangle_{0}$ is the " $\sigma$ "-condensate at $\mu_{I}=0$. This shows that the " $\sigma$ "-condensate decreases rapidly after the phase transition where the $\pi$-condensate grows rapidly. The " $\sigma$ "-condensate becomes very small for $\mu_{I} \gtrsim 400 \mathrm{MeV}$, while the $\pi$-condensate keeps increasing. Using the form $\left\langle\pi^{a}\right\rangle \propto\left(\mu_{I}-\mu_{I}^{c}\right)^{v}$ near the phase transition point, we fit the critical exponent $v$ to obtain $v=\frac{1}{2}$. This implies that the phase transition here is the mean field type.

We also show the "chiral circle" in Fig. 3 It is remarkable that the value of the "chiral condensate" defined by

$$
\langle\tilde{\sigma}\rangle=\sqrt{\langle\sigma\rangle^{2}+\left\langle\pi^{a}\right\rangle^{2}}
$$

is constant for increasing isospin chemical potential $\mu_{I}$ for $\mu_{I} \lesssim 300 \mathrm{MeV}$, and that it grows rapidly in the large $\mu_{I}$ region.

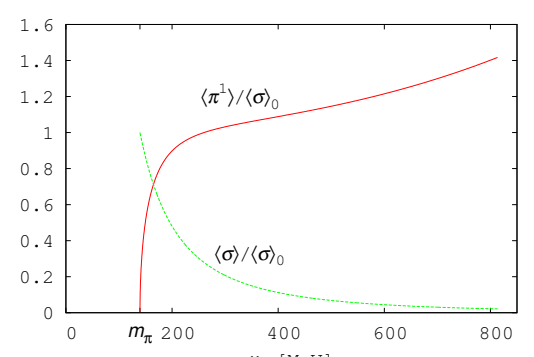

Figure 2: $\mu_{I}$ dependences of the $\pi$-condensate (red curve) and the " $\sigma$ "-condensate (green curve).

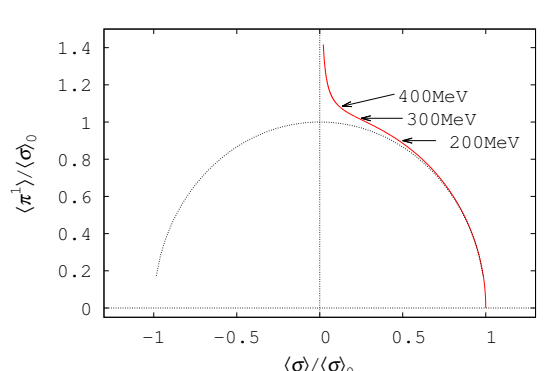

Figure 3: The chiral circle is showed as the red curve. The black curve is an unit circle.

\section{Comparison with the chiral Lagrangian}

In this section, we compare our result for the relation between the isospin number density $n_{I}$ and $\mu_{I}$ shown in Fig. 1 as well as the $\mu_{I}$-dependences of the $\pi$-condensate and the " $\sigma$ "-condensate in Fig. 2] with the ones from the chiral Lagrangian including the $\mathrm{O}\left(p^{4}\right)$ terms. Here we use the chiral Lagrangian for two flavor case [4]. One can simply introduce the isospin chemical potential $\mu_{I}$ as vacuum expectation values of these external gauge fields as $\left\langle\mathscr{L}_{\mu}^{3}\right\rangle=\left\langle\mathscr{R}_{\mu}^{3}\right\rangle=\frac{\mu_{I}}{2} \delta_{0 \mu}$ [3]. By minimizing the effective potential, we can obtain the relation between $n_{I}$ and $\mu_{I}, \frac{\langle\sigma\rangle}{\langle\sigma\rangle_{0}}$ and $\frac{\left\langle\pi^{1}\right\rangle}{\langle\sigma\rangle_{0}}$.

In Ref.[1, we fitted the values of the relevant low-energy constants in the chiral Lagrangian to our result on the $\mu_{I}$ dependence of $n_{I}, \frac{\langle\sigma\rangle}{\langle\sigma\rangle_{0}}$ and $\frac{\left\langle\pi^{1}\right\rangle}{\langle\sigma\rangle_{0}}$. We find that the deviation of our result from the one obtained from the $O\left(p^{2}\right)$ chiral Lagrangian is actually explained by including effects of $O\left(p^{4}\right)$ terms.

\section{A summary and discussions}

We studied the phase transition to the pion condensation phase for finite isospin chemical potential using the holographic QCD model given in Refs. [2], by introducding the isospin chemical potential $\mu_{I}$ as a UV-boundary value of the time component of the gauge field of $\mathrm{SU}(2)_{\mathrm{V}}$ symmetry as $\left.V_{0}^{3}(z)\right|_{\varepsilon}=\mu_{I}$. We assumed non-existence of vector meson condensates since we are interested 
in studying the small $\mu_{I}$ region. Furthermore, we assumed that the neutral pion does not condense. We solved the coupled equations of motion for the $\pi$-condensate and " $\sigma$ "-condensate together $V_{0}^{3}$ to determine $\mu_{I}$ as an eigenvalue.

Our result shows that the phase transition is of the second order and the critical chemical potential is predicted to be equal to the pion mass. This is consistent with the result obtained by the chiral Lagrangian approach in Ref. [3], but contrary to the result in Ref. [?]. Furthermore, our result on the relation between isospin number density and isospin chemical potential for small $\mu_{I}$ agrees with the following one obtained by $\mathrm{O}\left(p^{2}\right)$ chiral Lagrangian [3]. For large $\mu_{I}(>500 \mathrm{MeV})$, there is a difference between our predictions and the one from $\mathrm{O}\left(p^{2}\right)$ chiral Lagrangian, which is shown to be understood as the $\mathrm{O}\left(p^{4}\right)$ contributions.

We also studied the $\mu_{I}$ dependence of the $\pi$-condensate and " $\sigma$ "-condensate. Our result shows that, at the phase transition point, the $\pi$-condensate increases from zero with the mean field exponent Furthermore, we find that the " $\sigma$ "-condensate decreases rapidly after the phase transition where the $\pi$-condensate grows rapidly, while the value of the "chiral condensate" defined by $\langle\tilde{\sigma}\rangle=\sqrt{\langle\sigma\rangle^{2}+\left\langle\pi^{a}\right\rangle^{2}}$ is constant for $\mu_{I} \lesssim 300 \mathrm{MeV}$, and that it grows rapidly in the large $\mu_{I}$ region. This indicates that the chiral symmetry restoration at finite baryon density and/or finite temperature will be delayed when non-zero isospin chemical potential is turned on.

\section{Acknowledgements}

We would like to thank Shin Nakamura for useful discussions and comments. This work was supported in part by Grant-in-Aid for Scientific Research on Innovative Areas (No. 2104) "Quest on New Hadrons with Variety of Flavors" from MEXT, and by the JSPS Grant-in-Aid for Scientific Research (S) No. 22224003, (c) No. 24540266.

\section{References}

[1] H. Nishihara and M. Harada, arXiv:1401.2928 [hep-ph].

[2] J. Erlich et al, Phys. Rev. Lett. 95 (2005) 261602, L. D. Rold and A. Pomarol, Nuclear Physics B 721 (2005) 79-97, L. D. Rold and A. Pomarol, JHEP 0601 (2006) 157

[3] D. T. Son and M. A. Stephanov, Phys. Rev. Lett. 86 (2001) 592-595

[4] J. Gasser and H. Leutwyler, Nuclear Physics B 250 (1985) 465-516, M. Harada and K. Yamawaki, Phys. Rept. 381, 1 (2003). 